The Annals of Applied Probability

2000, Vol. 10, No. 2, 616-633

\title{
STEP SIZE CONTROL FOR THE UNIFORM APPROXIMATION OF SYSTEMS OF STOCHASTIC DIFFERENTIAL EQUATIONS WITH ADDITIVE NOISE
}

\author{
By Norbert Hofmann, ${ }^{1}$ Thomas MÜller-Gronbach and Klaus RitTer \\ Universität Erlangen-Nürnberg, Freie Universität Berlin \\ and Universität Passau
}

\begin{abstract}
We analyze the pathwise approximation for systems of stochastic differential equations. The pathwise distance between the solution and its approximation is measured globally on the unit interval in the $L_{\infty}$-norm, and we study the expectation of this distance. For systems with additive noise we obtain sharp lower and upper bounds for the minimal error in the class of arbitrary methods which use discrete observations of a Brownian path. The optimal order is achieved by an Euler scheme with adaptive stepsize control. We illustrate the superiority of the adaptive method compared to equidistant discretization by a simulation experiment.
\end{abstract}

1. Introduction. We study pathwise approximation for systems of stochastic differential equations. We consider numerical schemes that use the values of the driving Brownian motion at finitely many points and generate an approximation to the whole trajectory of the solution. Up to now, results are available mainly for equidistant discretizations or in terms of the maximal step size. Here we determine to what extent adaptive discretizations are superior to equidistant ones.

Let $X$ and $\bar{X}$ denote the exact solution and an approximate solution, respectively, of a given system of equations on $T=[0,1]$. For a corresponding pair of trajectories in $\mathbb{R}^{d}$ we define the error as

$$
\|X-\bar{X}\|_{\infty}=\max _{t \in T} \max _{1 \leq i \leq d}\left|X_{i}(t)-\bar{X}_{i}(t)\right|,
$$

that is, as the maximal distance of the components of $X$ and $\bar{X}$ on the interval $T$. Averaging over all trajectories we end up with the definition

$$
e(\bar{X})=\left(E\left(\|X-\bar{X}\|_{\infty}^{q}\right)\right)^{1 / q}, \quad 1 \leq q<\infty,
$$

of the error of $\bar{X}$.

For $n \in \mathbb{N}$ we use $\widehat{X}^{n}$ to denote the Euler scheme with constant step size $1 / n$. From Faure (1992) we know that

$$
e\left(\widehat{X}^{n}\right) \leq c(\ln n / n)^{1 / 2} .
$$

Received April 1999; revised September 1999.

${ }^{1}$ Supported in part by the DFG:GR 876/9-2.

AMS 1991 subject classifications. Primary 65U05; secondary 60H10.

Key words and phrases. Systems of stochastic differential equations, pathwise approximation, adaption, step-size control, asymptotic optimality. 
See also Bouleau and Lépingle [(1994), Remark 5.B.1.5].

We address the following questions: Is the upper bound in (1) sharp, and if so, what can be said about the asymptotic constants? Can we decrease the error by switching to a different discretization or approximation scheme? What is the minimal error that can be achieved by any method that uses $n$ observations of the driving Brownian motion?

We provide answers in an asymptotic sense for systems

$$
d X(t)=a(t, X(t)) d t+\sigma(t) d W(t)
$$

with additive noise. Here $W$ denotes an $m$-dimensional Brownian motion, and $a: T \times \mathbb{R}^{d} \rightarrow \mathbb{R}^{d}$ and $\sigma: T \rightarrow \mathbb{R}^{d \times m}$ satisfy certain smoothness conditions. For the best discretization the step size $\tau_{l+1}-\tau_{l}$ is proportional to $1 / \sigma_{*}^{2}\left(\tau_{l}\right)$, where

$$
\sigma_{*}(t)=\max _{1 \leq i \leq d}\left(\sum_{j=1}^{m} \sigma_{i, j}^{2}(t)\right)^{1 / 2} .
$$

It suffices to use the Euler scheme with this discretization to get an asymptotically optimal approximation scheme. The corresponding error is of order

$$
1 / \sqrt{2}\left\|\sigma_{*}\right\|_{2}(\ln n / n)^{1 / 2} .
$$

No method that uses $n$ observations of $W$ can lead to better results. The error of the Euler method with step size $1 / n$ differs from the above minimal error by the factor $\left\|\sigma_{*}\right\|_{\infty} /\left\|\sigma_{*}\right\|_{2}$.

Different results hold if the error is defined by the $L_{2}$-distance of $X$ and $\bar{X}$. In this case the best discretization takes steps proportional to $1 / \sigma^{*}\left(\tau_{l}\right)$ with

$$
\sigma^{*}(t)=\left(\sum_{i=1}^{d} \sum_{j=1}^{m} \sigma_{i, j}^{2}(t)\right)^{1 / 2} .
$$

See Hofmann, Müller-Gronbach and Ritter (1999a) for the case $d=m=$ 1. Furthermore, the minimal errors are of the order $n^{-1 / 2}$ and the diffusion coefficients enter the asymptotic constant of the minimal errors via $\left\|\sigma^{*}\right\|_{1}$. Hence there is a strong correspondence between the underlying norm and the best discretization. See Remark 1 for comments concerning $L_{p}$-approximation.

Now let us briefly discuss general systems,

$$
d X(t)=a(t, X(t)) d t+\sigma(t, X(t)) d W(t) .
$$

In Hofmann, Müller-Gronbach and Ritter (1999b), $L_{2}$-approximation for the case $d=m=1$ is analyzed comprehensively. Basically, a step size proportional to the inverse of the current value of $|\sigma|$ leads to an asymptotically optimal method. Moreover, at least some of the Euler steps must be replaced by Milstein steps. The results support our conjecture that for $L_{\infty}$-approximation of a general system (3) the best discretization selects the step size proportional to $\sigma_{*}^{-2}$. Observe that in both cases the step size is adapted to every single trajectory, since $\sigma$ depends on the state variable, too. 
We add that discrete norms, that is, the distance between $X$ and $\bar{X}$ at a finite number of points, are frequently used in the literature. See Hofmann, Müller-Gronbach and Ritter (1999a) for a discussion. Only a few papers deal with adaptive step size control. Our approach is related to Cambanis and $\mathrm{Hu}$ (1996), where the mean square error at the point $t=1$ is studied. Different approaches towards adaptive step size control are taken in Newton (1990), Gaines and Lyons (1997) and Mauthner (1998).

The structure of the paper is as follows. In Section 2 we introduce the adaptive discretization and present the asymptotic analysis for the corresponding Euler scheme. We provide the asymptotic result for the Euler scheme with constant step size for comparison. Moreover, we justify the new method by a matching lower bound for the class of arbitrary methods that use a finite number of observations of the driving Brownian motion. In Section 3 we report a simulation experiment that confirms the theoretical results. Proofs are given in Section 4. The Appendix contains an upper bound for the error of the Euler method, which is a helpful tool in our analysis.

2. Main results. The system (2) consists of the equations

$$
d X_{i}(t)=a_{i}(t, X(t)) d t+\sum_{j=1}^{m} \sigma_{i, j}(t) d W_{j}(t)
$$

for $t \in T$ and $i=1, \ldots, d$, and it is driven by an $m$-dimensional Brownian motion $W=\left(W_{1}, \ldots, W_{m}\right)$ on the unit interval $T$. The Euler scheme for (4) is based on a discretization

$$
0=\tau_{0}<\cdots<\tau_{n}=1
$$

of the unit interval. A discrete approximation for the solution $X$ is defined by

$$
\widehat{X}_{i}\left(\tau_{0}\right)=X_{i}(0)
$$

for $i=1, \ldots, d$ with $X(0)$ denoting the vector of initial values, and

$$
\widehat{X}_{i}\left(\tau_{l+1}\right)=\widehat{X}_{i}\left(\tau_{l}\right)+a_{i}\left(\tau_{l}, \widehat{X}\left(\tau_{l}\right)\right)\left(\tau_{l+1}-\tau_{l}\right)+\sum_{j=1}^{m} \sigma_{i, j}\left(\tau_{l}\right)\left(W_{j}\left(\tau_{l+1}\right)-W_{j}\left(\tau_{l}\right)\right)
$$

for $l=0, \ldots, n-1$ and $i=1, \ldots, d$. A global approximation $\widehat{X}$ for $X$ on $T$ is obtained by piecewise linear interpolation of the data $\left(\tau_{l}, \widehat{X}\left(\tau_{l}\right)\right)$.

We suggest using the following adaptive step size control for the Euler scheme in order to obtain small errors in $L_{\infty}$-norm. Choose a basic step size,

$$
h \in] 0,1[\text {, }
$$

put $\tau_{0}=0$, and define

$$
\tau_{l+1}=\tau_{l}+h / \sigma_{*}^{2}\left(\tau_{l}\right)
$$

as long as the right-hand side does not exceed 1 . Otherwise, put $\tau_{l+1}=1$. We use $\widehat{X}^{h}$ to denote the corresponding Euler scheme in the sequel. 
By $|\cdot|_{\infty}$ we denote the $\ell_{\infty}$-norm on the space $\mathbb{R}^{d}$ and by $\|\cdot\|_{p}$ the $L_{p}$-norm of real-valued functions on $T$. We define $\|f\|_{p}=\max _{1 \leq i \leq d}\left\|f_{i}\right\|_{p}$ for $\mathbb{R}^{d}$-valued functions $f$ on $T$.

We assume that the drift vector $a: T \times \mathbb{R}^{d} \rightarrow \mathbb{R}^{d}$ and the diffusion matrix $\sigma: T \rightarrow \mathbb{R}^{d \times m}$ have the following properties.

(A) There exist constants $K_{1}, K_{2}, K_{3}>0$ such that

$$
|a(t, x)-a(t, y)|_{\infty} \leq K_{1}|x-y|_{\infty}, \quad|a(t, 0)|_{\infty} \leq K_{2}
$$

and

$$
|a(t, x)-a(s, x)|_{\infty} \leq K_{3}\left(1+|x|_{\infty}\right)|t-s|^{1 / 2}
$$

for all $s, t \in T$ and $x, y \in \mathbb{R}^{d}$.

(B) $\sigma$ is continuously differentiable with

$$
\sigma_{*}(t)>0
$$

for all $t \in T$, and there exist constants $K_{4}, K_{5}>0$ such that

$$
\left\|\sigma_{i, j}\right\|_{\infty} \leq K_{4}, \quad\left\|\sigma_{i, j}^{\prime}\right\|_{\infty} \leq K_{5}
$$

for all $i=1, \ldots, d$ and $j=1, \ldots, m$.

Furthermore, we assume that

(C) $X(0)$ is independent of $W$ and

$$
E\left(|X(0)|_{\infty}^{q}\right) \leq K_{6}
$$

for some constant $K_{6}>0$.

Conditions (A) and (C) are standard assumptions for analyzing stochastic differential systems, while (B) is slightly stronger than the standard assumption for systems with additive noise. See, for example, Bouleau and Lépingle [(1994), Chapter 5]. In particular, the conditions assure that

$$
E\left(\|X\|_{\infty}^{q}\right)<\infty
$$

At first we compare equidistant discretization and our adaptive discretization (6) for the Euler scheme. We use $n\left(h, \sigma_{*}\right)$ to denote the total number of discretization points in (6).

THEOREM 1. Assume that (A)-(C) hold for the system of equations (4). Then

$$
\lim _{h \rightarrow 0}\left(n\left(h, \sigma_{*}\right) / \ln n\left(h, \sigma_{*}\right)\right)^{1 / 2} e\left(\widehat{X}^{h}\right)=1 / \sqrt{2}\left\|\sigma_{*}\right\|_{2}
$$

for the Euler method based on the discretization (6). The Euler approximation $\widehat{X}^{n}$ with constant step-size

$$
\tau_{k+1}-\tau_{k}=1 / n
$$

yields

$$
\lim _{n \rightarrow \infty}(n / \ln n)^{1 / 2} e\left(\widehat{X}^{n}\right)=1 / \sqrt{2}\left\|\sigma_{*}\right\|_{\infty} .
$$


Taking (6) instead of an equidistant discretization therefore reduces the error roughly by the factor

$$
\left\|\sigma_{*}\right\|_{2} /\left\|\sigma_{*}\right\|_{\infty}
$$

for the same number of observations of $W$. The overall computational cost increases by just a few arithmetical operations per step, which is irrelevant in practice.

Choosing steps of length $h / \sigma_{*}^{2}\left(\tau_{l}\right)$ leads to the good asymptotic performance of $\widehat{X}^{h}$. However, we want to control the error for larger values of $h$ or small values of $\sigma_{*}\left(\tau_{l}\right)$. To this end we modify (6) according to

$$
\tau_{l+1}=\tau_{l}+\min \left(h \ln h^{-1}, h / \sigma_{*}^{2}\left(\tau_{l}\right)\right) .
$$

See Remark 2 for details. A step of size $h \ln h^{-1}$ is only taken, if $h>$ $\exp \left(-\sigma_{*}^{-2}\left(\tau_{l}\right)\right)$. Obviously the additional term is irrelevant asymptotically.

Now we show that no other discretization or other use of the data $W\left(\tau_{1}\right), \ldots, W\left(\tau_{n}\right)$ and $X(0)$ can lead to better asymptotic results. An arbitrary method $\bar{X}^{n}$ with components $\bar{X}_{i}^{n}$ that is based on a realization of the vector of initial values $X(0)$ and on $n$ observations of a trajectory of the $m$-dimensional Brownian motion $W$ is defined by measurable mappings

$$
\psi_{k}: \mathbb{R}^{(k-1) m+d} \rightarrow T
$$

for $k=1, \ldots, n$ and

$$
\phi_{i}: \mathbb{R}^{n m+d} \rightarrow L_{\infty}(T)
$$

for $i=1, \ldots, d$. The mapping $\psi_{k}$ determines the observation point in step $k$ in terms of the previous evaluations. A pathwise approximation is computed according to

$$
\bar{X}_{i}^{n}=\phi_{i}\left(X(0), Y_{1}, \ldots, Y_{n}\right),
$$

for $i=1, \ldots, d$, where $Y_{1}=W\left(\psi_{1}(X(0))\right)$ and

$$
Y_{k}=W\left(\psi_{k}\left(X(0), Y_{1}, \ldots, Y_{k-1}\right)\right)
$$

is the observation in step $k \geq 2$. Every such method is called an $n$-point method in the sequel.

The quantity

$$
e(n)=\inf _{\bar{X}^{n}} e\left(\bar{X}^{n}\right)
$$

is the minimal error that can be obtained by $n$-point methods for the system of equations (4).

THEOREM 2. Assume that (A)-(C) hold for the system of equations (4). The minimal errors satisfy

$$
\lim _{n \rightarrow \infty}(n / \ln n)^{1 / 2} e(n)=1 / \sqrt{2}\left\|\sigma_{*}\right\|_{2} .
$$


Due to Theorems 1 and 2, the Euler method $\widehat{X}^{h}$ is asymptotically optimal for every system of equations with additive noise.

REMARK 1. Our approach also covers the asymptotic analysis of errors

$$
e(\bar{X})=\left(E\left(\|X-\bar{X}\|_{p}^{p}\right)\right)^{1 / p}, \quad 1 \leq p<\infty .
$$

For scalar equations with additive noise we propose an adaptive discretization that takes steps proportionally to $1 / \sigma^{\lambda}\left(\tau_{l}\right)$ with $\lambda=1 /\left(2^{-1}+p^{-1}\right)$. The error of the corresponding Euler scheme $\widehat{X}$ asymptotically behaves like $\left(\eta_{p} \mu_{p}\right)^{1 / p}\|\sigma\|_{\lambda} n^{-1 / 2}$, which is best possible.

Here $\eta_{p}$ denotes the $p$ th moment of the absolute value of a standard Gaussian random variable and $\mu_{p}=\Gamma^{2}(p / 2+1) / \Gamma(p+2)$. For the Euler approximation with constant step size $1 / n$ we get the asymptotic constant $\left(\eta_{p} \mu_{p}\right)^{1 / p}\|\sigma\|_{p}$.

These statements can be proved by a straightforward generalization of the method from Hofmann, Müller-Gronbach and Ritter (1999a).

3. Simulation experiment. We consider the scalar equation

$$
d X(t)=-2 d t+\sigma(t) d W(t), \quad X(0)=1,
$$

driven by a one-dimensional Brownian motion $W$. We choose the diffusion coefficient $\sigma$ according to

$$
\sigma(t)=20 \exp \left(-(10 t-1)^{2}\right)-t .
$$

The solution of (9) is not known explicitly. Instead of $X$ we therefore use an $M$-point Euler approximation $\widehat{X}_{M}$ with $M$ sufficiently large. We simulate $K=10000$ trajectories of $W$ and use

$$
\varepsilon(\bar{X})=\left(\frac{1}{K} \sum_{k=1}^{K}\left\|\widehat{X}_{M}\left(\omega_{k}\right)-\bar{X}\left(\omega_{k}\right)\right\|_{\infty}^{q}\right)^{1 / q}
$$

as an estimator for $e(\bar{X})$. The quantities $\varepsilon\left(\widehat{X}^{h}\right)$ and $\varepsilon\left(\widehat{X}^{n}\right)$ are easy to compute. We express the efficiency of the method $\widehat{X}^{h}$ by the ratio

$$
\operatorname{eff}(h)=\varepsilon\left(\widehat{X}^{h}\right) / \varepsilon\left(\widehat{X}^{n}\right) .
$$

Theorem 1 tells us that $\operatorname{eff}(h)$ is approximately given by

$$
\|\sigma\|_{2} /\|\sigma\|_{\infty}=0.35 \ldots
$$

for sufficiently large $K$ and small $h$. We also show the mean ratio ex $(h)$ between steps of length $h \ln h^{-1}$ (called exceptions) and the total number of steps in Table 1.

The same equation is studied in Hofmann, Müller-Gronbach and Ritter (1999a), where pathwise approximation with respect to the global error in the $L_{2}$-norm is analyzed. A comparison of the results shows a much slower convergence of the efficiency in the $L_{\infty}$-case. The proofs and a closer look to 
TABLE 1

Simulation for (9) and $q=2$ with different basic step sizes $h$

\begin{tabular}{lccccc}
\hline $\boldsymbol{h}$ & $\boldsymbol{\varepsilon}\left(\widehat{\boldsymbol{X}}^{\boldsymbol{h}}\right)$ & $\boldsymbol{n}(\boldsymbol{h}, \boldsymbol{\sigma})$ & $\boldsymbol{\varepsilon}\left(\widehat{\boldsymbol{X}}^{\boldsymbol{n}}\right)$ & $\operatorname{eff}(\boldsymbol{h})$ & $\operatorname{ex}(\boldsymbol{h})$ \\
\hline $5 \cdot 10^{-1}$ & 1.246034 & 100 & 2.489485 & 0.50 & 0.0200 \\
$1 \cdot 10^{-1}$ & 0.651305 & 489 & 1.262255 & 0.52 & 0.0041 \\
$5 \cdot 10^{-2}$ & 0.485591 & 975 & 0.954914 & 0.51 & 0.0021 \\
$1 \cdot 10^{-2}$ & 0.242423 & 4864 & 0.487348 & 0.50 & 0.0008 \\
$5 \cdot 10^{-3}$ & 0.170084 & 9725 & 0.360682 & 0.47 & 0.0006 \\
$1 \cdot 10^{-3}$ & 0.075044 & 48608 & 0.179113 & 0.42 & 0.0003 \\
$5 \cdot 10^{-4}$ & 0.054014 & 97212 & 0.131334 & 0.41 & 0.0002 \\
\hline
\end{tabular}

the data reveal that this is caused by the error $\varepsilon\left(\widehat{X}^{n}\right)$ of the Euler method with equidistant discretization. In contrast to the $L_{2}$-case the asymptotical behavior of $e\left(\widehat{X}^{n}\right)$ is now determined by the performance of $\widehat{X}^{n}$ around the points where $\sigma_{*}$ takes its maximum. Therefore, a large number of discretization points is needed around these sites, and the total number of points has to be very large.

4. Proofs. The proofs are based on the decomposition

$$
X(t)=X(0)+V(t)+Y(t)-Z(t)
$$

with components

$$
\begin{aligned}
& V_{i}(t)=\sum_{j=1}^{m} \sigma_{i, j}(t) W_{j}(t), \\
& Y_{i}(t)=\int_{0}^{t} a_{i}(s, X(s)) d s
\end{aligned}
$$

and

$$
Z_{i}(t)=\int_{0}^{t} \sum_{j=1}^{m} \sigma_{i, j}^{\prime}(s) W_{j}(s) d s
$$

for $i=1, \ldots, d$. Fix a discretization (5) and let

$$
\Delta_{l}=\tau_{l}-\tau_{l-1}
$$

and

$$
\Delta_{\max }=\max _{1 \leq l \leq n} \Delta_{l} .
$$

The corresponding Euler approximation $\widehat{X}$ of $X$ may be written as

$$
\widehat{X}\left(\tau_{l}\right)=X(0)+\widehat{V}\left(\tau_{l}\right)+\widehat{Y}\left(\tau_{l}\right)-\widehat{Z}\left(\tau_{l}\right)
$$


with components

$$
\begin{aligned}
\widehat{V}_{i}\left(\tau_{l}\right) & =\sum_{j=1}^{m} \sigma_{i, j}\left(\tau_{l}\right) W_{j}\left(\tau_{l}\right) \\
\widehat{Y}_{i}\left(\tau_{l}\right) & =\sum_{k=1}^{l} a_{i}\left(\tau_{k-1}, \widehat{X}\left(\tau_{k-1}\right)\right) \Delta_{k}
\end{aligned}
$$

and

$$
\widehat{Z}_{i}\left(\tau_{l}\right)=\sum_{k=1}^{l}\left(\sum_{j=1}^{m}\left(\sigma_{i, j}\left(\tau_{k}\right)-\sigma_{i, j}\left(\tau_{k-1}\right)\right) W_{j}\left(\tau_{k}\right)\right)
$$

for $i=1, \ldots, d$. Piecewise linear interpolation yields $\mathbb{R}^{d}$-valued processes $\widehat{V}$, $\widehat{Y}$ and $\widehat{Z}$ over $T$.

4.1. Preliminary estimates. In the sequel $\tilde{X}$ denotes the piecewise linear interpolation of an arbitrary $\mathbb{R}^{d}$-valued process $X$ on $T$. Thus

$$
\tilde{X}(t)=\left(\left(t-\tau_{l-1}\right) X\left(\tau_{l}\right)+\left(\tau_{l}-t\right) X\left(\tau_{l-1}\right)\right) / \Delta_{l}
$$

for $t \in\left[\tau_{l-1}, \tau_{l}\right]$. Moreover, we use $c$ to denote unspecified positive constants which only depend on $q, d, m$ and on the constants from conditions (A)-(C).

At first we compare the processes $Y$ and $Z$ with their counterparts $\widehat{Y}$ and $\widehat{Z}$ from the corresponding Euler approximation.

LEMMA 1. We have

$$
\left(E\left(\|Z-\widehat{Z}\|_{\infty}^{q}\right)\right)^{1 / q} \leq c \Delta_{\max }
$$

and

$$
\left(E\left(\|Y-\widehat{Y}\|_{\infty}^{q}\right)\right)^{1 / q} \leq c \Delta_{\max }^{1 / 2}
$$

PROOF. Let $t \in\left[\tau_{l-1}, \tau_{l}\right]$. Observing property (B) we get

$$
\begin{aligned}
\left|Z_{i}(t)-\widetilde{Z}_{i}(t)\right| & \leq\left(\left(\tau_{l}-t\right)\left|Z_{i}(t)-Z_{i}\left(\tau_{l-1}\right)\right|+\left(t-\tau_{l-1}\right)\left|Z_{i}(t)-Z_{i}\left(\tau_{l}\right)\right|\right) / \Delta_{l} \\
& \leq \int_{\tau_{l-1}}^{\tau_{l}} \sum_{j=1}^{m}\left|\sigma_{i, j}^{\prime}(s) W_{j}(s)\right| d s \\
& \leq K_{5} \Delta_{l} \sum_{j=1}^{m}\left\|W_{j}\right\|_{\infty} .
\end{aligned}
$$

Therefore,

$$
\left(E\left(\left\|Z_{i}-\widetilde{Z}_{i}\right\|_{\infty}^{q}\right)\right)^{1 / q} \leq c \Delta_{\max }
$$


Clearly,

$$
\left\|\widetilde{Z}_{i}-\widehat{Z}_{i}\right\|_{\infty}=\max _{1 \leq l \leq n}\left|\sum_{k=1}^{l} \int_{\tau_{k-1}}^{\tau_{k}} \sum_{j=1}^{m} \sigma_{i, j}^{\prime}(s)\left(W_{j}(s)-W_{j}\left(\tau_{k}\right)\right) d s\right| .
$$

We use Lévy's inequality and (B) to obtain

$$
\begin{aligned}
(E & \left.\left(\left\|\widetilde{Z}_{i}-\widehat{Z}_{i}\right\|_{\infty}^{q}\right)\right)^{1 / q} \\
& \leq 2^{1 / q}\left(E\left(\left|\sum_{k=1}^{n} \int_{\tau_{k-1}}^{\tau_{k}} \sum_{j=1}^{m} \sigma_{i, j}^{\prime}(s)\left(W_{j}(s)-W_{j}\left(\tau_{k}\right)\right) d s\right|^{q}\right)\right)^{1 / q} \\
& =c\left(E\left(\left|\sum_{k=1}^{n} \int_{\tau_{k-1}}^{\tau_{k}} \sum_{j=1}^{m} \sigma_{i, j}^{\prime}(s)\left(W_{j}(s)-W_{j}\left(\tau_{k}\right)\right) d s\right|^{2}\right)\right)^{1 / 2} \\
& \leq c\left(\sum_{k=1}^{n} \int_{\tau_{k-1}}^{\tau_{k}} \int_{\tau_{k-1}}^{\tau_{k}}\left(\tau_{k}-\max (s, t)\right) d s d t\right)^{1 / 2} \\
& \leq c\left(\sum_{k=1}^{n} \Delta_{k}^{3}\right)^{1 / 2} \\
& \leq c \Delta_{\max } \cdot
\end{aligned}
$$

Now (11), (12) and

$$
\left(E\left(\|Z-\widehat{Z}\|_{\infty}^{q}\right)\right)^{1 / q} \leq \sum_{i=1}^{d}\left(E\left(\left\|Z_{i}-\widehat{Z}_{i}\right\|_{\infty}^{q}\right)\right)^{1 / q}
$$

yield the first estimate.

Due to condition (A) we have

$$
\left\|Y_{i}-\tilde{Y}_{i}\right\|_{\infty} \leq \max _{1 \leq l \leq n} \int_{\tau_{l-1}}^{\tau_{l}}\left|a_{i}(s, X(s))\right| d s \leq c \Delta_{\max }\left(1+\|X\|_{\infty}\right) .
$$

Therefore,

$$
\left(E\left(\left\|Y_{i}-\tilde{Y}_{i}\right\|_{\infty}^{q}\right)\right)^{1 / q} \leq c \Delta_{\max }
$$

by (7). Furthermore,

$$
\begin{aligned}
\left\|\tilde{Y}_{i}-\widehat{Y}_{i}\right\|_{\infty} & =\max _{1 \leq l \leq n}\left|Y_{i}\left(\tau_{l}\right)-\widehat{Y}_{i}\left(\tau_{l}\right)\right| \\
& \leq \max _{1 \leq l \leq n}\left|X_{i}\left(\tau_{l}\right)-\widehat{X}_{i}\left(\tau_{l}\right)\right|+\max _{1 \leq l \leq n}\left|Z_{i}\left(\tau_{l}\right)-\widehat{Z}_{i}\left(\tau_{l}\right)\right|,
\end{aligned}
$$

together with (32) in the Appendix and the first estimate, yields

$$
\left(E\left(\left\|\tilde{Y}_{i}-\widehat{Y}_{i}\right\|_{\infty}^{q}\right)\right)^{1 / q} \leq c \Delta_{\max }^{1 / 2}+c \Delta_{\max } \leq c \Delta_{\max }^{1 / 2} .
$$


Combine (13), (14) and

$$
\left(E\left(\|Y-\widehat{Y}\|_{\infty}^{q}\right)\right)^{1 / q} \leq \sum_{i=1}^{d}\left(E\left(\left\|Y_{i}-\widehat{Y}_{i}\right\|_{\infty}^{q}\right)\right)^{1 / q}
$$

to complete the proof.

We relate the error of the Euler method to a weighted $L_{\infty}$-error of piecewise linear interpolation of $W$.

LEMMA 2.

$$
\left|e(\widehat{X})-\left(E\left(\|\sigma(W-\widetilde{W})\|_{\infty}^{q}\right)\right)^{1 / q}\right| \leq c \Delta_{\max }^{1 / 2}
$$

For the proof, use (B) and Lemma 1.

4.2. Proof of the lower bounds in Theorem 1. In the sequel we use $B_{1}, B_{2}, \ldots$ to denote independent Brownian bridges on $T$ with $B_{l}(0)=$ $B_{l}(1)=0$, and we put

$$
B_{l}^{*}=\left\|B_{l}\right\|_{\infty} .
$$

Moreover, let

$$
\sigma_{i}(t)=\left(\sum_{j=1}^{m} \sigma_{i, j}(t)^{2}\right)^{1 / 2}
$$

such that

$$
\sigma_{*}(t)=\max _{1 \leq i \leq d} \sigma_{i}(t)
$$

Take $i \in\{1, \ldots, d\}$ with

$$
\left\|\sigma_{i}\right\|_{\infty}=\left\|\sigma_{*}\right\|_{\infty}
$$

Clearly

$$
\left(E\left(\|\sigma(W-\tilde{W})\|_{\infty}^{q}\right)\right)^{1 / q} \geq\left(E\left(\left\|\sigma_{i}\left(W_{1}-\tilde{W}_{1}\right)\right\|_{\infty}^{q}\right)\right)^{1 / q} .
$$

Moreover,

$$
\begin{aligned}
& \left(E\left(\left\|\sigma_{i}\left(W_{1}-\tilde{W}_{1}\right)\right\|_{\infty}^{q}\right)\right)^{1 / q} \\
& \quad=\left(E\left(\max _{1 \leq l \leq n}\left(\Delta_{l}^{1 / 2} \max _{\tau_{l-1} \leq t \leq \tau_{l}}\left|\sigma_{i}(t) B_{l}\left(\left(t-\tau_{l-1}\right) / \Delta_{l}\right)\right|\right)^{q}\right)\right)^{1 / q},
\end{aligned}
$$


and therefore,

$$
\begin{aligned}
& \left|\left(E\left(\left\|\sigma_{i}\left(W_{1}-\tilde{W}_{1}\right)\right\|_{\infty}^{q}\right)\right)^{1 / q}-\left(E\left(\max _{1 \leq l \leq n}\left(\Delta_{l}^{1 / 2} \sigma_{i}\left(\tau_{l-1}\right) B_{l}^{*}\right)^{q}\right)\right)^{1 / q}\right| \\
& \quad \leq c \Delta_{\max }
\end{aligned}
$$

by property (B). Due to Lemma 2 as well as (15) and (16) we have

$$
e(\widehat{X}) \geq\left(E\left(\max _{1 \leq l \leq n}\left(\Delta_{l}^{1 / 2} \sigma_{i}\left(\tau_{l-1}\right) B_{l}^{*}\right)^{q}\right)\right)^{1 / q}-c \Delta_{\max }^{1 / 2} .
$$

Now we consider the particular discretization (6). Clearly $\sigma_{*}\left(\tau_{l-1}\right) \Delta_{l}^{1 / 2}=$ $h^{1 / 2}$ and

$$
\Delta_{\max } \leq\left\|\sigma_{*}^{-2}\right\|_{\infty} h
$$

From (17) we get

$$
e\left(\widehat{X}^{h}\right) \geq h^{1 / 2}\left(E\left(\max _{1 \leq l \leq n}\left(\sigma_{i}\left(\tau_{l-1}\right) / \sigma_{*}\left(\tau_{l-1}\right) B_{l}^{*}\right)^{q}\right)\right)^{1 / q}-c\left\|\sigma_{*}^{-2}\right\|_{\infty} h^{1 / 2} .
$$

Moreover,

$$
\lim _{h \rightarrow 0} n\left(h, \sigma_{*}\right) h=\left\|\sigma_{*}\right\|_{2}^{2}
$$

Corollary 1 in the Appendix yields

$$
\begin{aligned}
& \liminf _{h \rightarrow 0}\left(n\left(h, \sigma_{*}\right) / \ln n\left(h, \sigma_{*}\right)\right)^{1 / 2} e\left(\widehat{X}^{h}\right) \\
& \geq \liminf _{h \rightarrow 0}\left(n\left(h, \sigma_{*}\right) h\right)^{1 / 2}\left(\ln n\left(h, \sigma_{*}\right)\right)^{-1 / 2} \\
& \quad \times\left(E\left(\max _{1 \leq l \leq n\left(h, \sigma_{*}\right)}\left(\left(\sigma_{i}\left(\tau_{l-1}\right) / \sigma_{*}\left(\tau_{l-1}\right)\right) B_{l}^{*}\right)^{q}\right)\right)^{1 / q} \\
& =\left\|\sigma_{*}\right\|_{2} 2^{-1 / 2} .
\end{aligned}
$$

Next we consider the constant step size

$$
\Delta_{l}=1 / n \text {. }
$$

We apply Corollary 1 in the Appendix and (17) to obtain

$$
\begin{aligned}
& \liminf _{n \rightarrow \infty}(n / \ln n)^{1 / 2} e\left(\widehat{X}^{n}\right) \\
& \quad \geq \liminf _{n \rightarrow \infty}(\ln n)^{-1 / 2}\left(E\left(\max _{1 \leq l \leq n}\left(\sigma_{i}((l-1) / n) B_{l}^{*}\right)^{q}\right)\right)^{1 / q} \\
& \quad=\left\|\sigma_{*}\right\|_{\infty} 2^{-1 / 2} .
\end{aligned}
$$


4.3. Proof of the upper bounds in Theorem 1. Due to Lemma 2 as well as (18) and (19) it suffices to study $\|\sigma(W-\widetilde{W})\|_{\infty}$. Put $\sigma_{d+1,1}=\sigma_{*}$ and $\sigma_{d+1, j}=0$ for $j=2, \ldots, m$. Note that

$$
\begin{aligned}
(n / \ln n)^{q / 2} E\left(\|\sigma(W-\widetilde{W})\|_{\infty}^{q}\right) & \\
\leq & \sum_{i=1}^{d+1} \int_{z}^{\infty} P\left(\left\|(n / \ln n)^{1 / 2} \sum_{j=1}^{m} \sigma_{i, j}\left(W_{j}-\widetilde{W}_{j}\right)\right\|_{\infty}^{q}>u\right) d u \\
& +\int_{0}^{z} P\left(\max _{1 \leq i \leq d+1}\left\|(n / \ln n)^{1 / 2} \sum_{j=1}^{m} \sigma_{i, j}\left(W_{j}-\tilde{W}_{j}\right)\right\|_{\infty}^{q}>u\right) d u
\end{aligned}
$$

for every $z>0$. Moreover,

$$
\begin{aligned}
P\left(\left\|\sum_{j=1}^{m} \sigma_{i, j}\left(W_{j}-\tilde{W}_{j}\right)\right\|_{\infty}>v\right) & =P\left(\left\|\sigma_{i}\left(W_{1}-\widetilde{W}_{1}\right)\right\|_{\infty}>v\right) \\
& \leq P\left(\left\|\sigma_{*}\left(W_{1}-\tilde{W}_{1}\right)\right\|_{\infty}>v\right) \\
& \leq P\left(\max _{1 \leq l \leq n} \max _{l-1 \leq t \leq \tau_{l}}\left(\sigma_{*}(t) \Delta_{l}^{1 / 2} B_{l}^{*}\right)>v\right)
\end{aligned}
$$

as well as

$$
\begin{gathered}
P\left(\max _{1 \leq i \leq d+1}\left\|\sum_{j=1}^{m} \sigma_{i, j}\left(W_{j}-\widetilde{W}_{j}\right)\right\|_{\infty}>v\right) \\
\geq P\left(\left\|\sum_{j=1}^{m} \sigma_{d+1, j}\left(W_{j}-\widetilde{W}_{j}\right)\right\|_{\infty}>v\right) \\
=P\left(\left\|\sigma_{*}\left(W_{1}-\tilde{W}_{1}\right)\right\|_{\infty}>v\right) \\
\geq P\left(\max _{1 \leq l \leq n} \min _{l-1 \leq t \leq \tau_{l}}\left(\sigma_{*}(t) \Delta_{l}^{1 / 2} B_{l}^{*}\right)>v\right)
\end{gathered}
$$

for every $v>0$.

First we consider the discretization (6). Put $n=n\left(h, \sigma_{*}\right)$ to obtain

$$
\begin{aligned}
& P\left(\left\|(n / \ln n)^{1 / 2} \sum_{j=1}^{m} \sigma_{i, j}\left(W_{j}-\tilde{W}_{j}\right)\right\|_{\infty}^{q}>u\right) \\
& \quad \leq P\left(\max _{1 \leq l \leq n}\left((n h)^{1 / 2} \frac{\max _{\tau_{l-1} \leq t \leq \tau_{l}} \sigma_{*}(t)}{\sigma_{*}\left(\tau_{l-1}\right)}(\ln n)^{-1 / 2} B_{l}^{*}\right)^{q}>u\right)
\end{aligned}
$$


from (21). Analogously, (22) yields

$$
\begin{aligned}
& P\left(\max _{1 \leq i \leq d+1}\left\|(n / \ln n)^{1 / 2} \sum_{j=1}^{m} \sigma_{i, j}\left(W_{j}-\widetilde{W}_{j}\right)\right\|_{\infty}^{q}>u\right) \\
& \quad \geq P\left(\max _{1 \leq l \leq n}\left((n h)^{1 / 2} \frac{\min _{\tau_{l-1} \leq t \leq \tau_{l}} \sigma_{*}(t)}{\sigma_{*}\left(\tau_{l-1}\right)}(\ln n)^{-1 / 2} B_{l}^{*}\right)^{q}>u\right) .
\end{aligned}
$$

Observing (19), we take

$$
z=\left(\left\|\sigma_{*}\right\|_{2} / 2^{1 / 2}\right)^{q}
$$

in (20) and apply Lemma 3 in the Appendix with $\alpha=\left\|\sigma_{*}\right\|_{2}$ to obtain

$$
\limsup _{h \rightarrow 0}(n / \ln n)^{q / 2} E\left(\|\sigma(W-\widetilde{W})\|_{\infty}^{q}\right) \leq\left(\left\|\sigma_{*}\right\|_{2} / 2^{1 / 2}\right)^{q} .
$$

For an equidistant discretization we get

$$
\begin{aligned}
& P\left(\left\|(n / \ln n)^{1 / 2} \sum_{j=1}^{m} \sigma_{i, j}\left(W_{j}-\tilde{W}_{j}\right)\right\|_{\infty}^{q}>u\right) \\
& \quad \leq P\left(\max _{1 \leq l \leq n}\left(\max _{\tau_{l-1} \leq t \leq \tau_{l}} \sigma_{*}(t)(\ln n)^{-1 / 2} B_{l}^{*}\right)^{q}>u\right)
\end{aligned}
$$

from (21) and

$$
\begin{gathered}
P\left(\max _{1 \leq i \leq d+1}\left\|(n / \ln n)^{1 / 2} \sum_{j=1}^{m} \sigma_{i, j}\left(W_{j}-\tilde{W}_{j}\right)\right\|_{\infty}^{q}>u\right) \\
\geq P\left(\max _{1 \leq l \leq n}\left(\min _{\tau_{l-1} \leq t \leq \tau_{l}} \sigma_{*}(t)(\ln n)^{-1 / 2} B_{l}^{*}\right)^{q}>u\right)
\end{gathered}
$$

from (22). We take

$$
z=\left(\left\|\sigma_{*}\right\|_{\infty} / 2^{1 / 2}\right)^{q}
$$

in (20) and apply Lemma 3 in the Appendix with $\alpha=\left\|\sigma_{*}\right\|_{\infty}$ to obtain

$$
\limsup _{n \rightarrow \infty}(n / \ln n)^{q / 2} E\left(\|\sigma(W-\tilde{W})\|_{\infty}^{q}\right) \leq\left(\left\|\sigma_{*}\right\|_{\infty} / 2^{1 / 2}\right)^{q} .
$$

This completes the proof of the upper bounds in Theorem 1.

REMARK 2. Consider the Euler method with an arbitrary discretization (5).

From Lemma 2 we get

$$
e(\widehat{X}) \leq c\left(\left(E\left(\left\|\sigma_{*}\left(W_{1}-\tilde{W}_{1}\right)\right\|_{\infty}^{q}\right)\right)^{1 / q}+\Delta_{\max }^{1 / 2}\right)
$$


as a rough estimate. Assumption (B) and Corollary 1 in the Appendix imply

$$
\begin{aligned}
& \left(E\left(\left\|\sigma_{*}\left(W_{1}-\widetilde{W}_{1}\right)\right\|_{\infty}^{q}\right)\right)^{1 / q} \\
& \quad \leq\left(E\left(\max _{1 \leq l \leq n}\left(\left(\sigma_{*}\left(\tau_{l-1}\right)+c \Delta_{l}\right) \Delta_{l}^{1 / 2} B_{l}^{*}\right)^{q}\right)\right)^{1 / q} \\
& \quad \leq c \max _{1 \leq l \leq n}\left(\sigma_{*}\left(\tau_{l-1}\right)+c \Delta_{l}\right) \Delta_{l}^{1 / 2}(\ln n)^{1 / 2}
\end{aligned}
$$

If $\Delta_{l}=h / \sigma_{*}^{2}\left(\tau_{l-1}\right)$, then $n\left(h, \sigma_{*}\right) \leq c / h$ due to (B), and we obtain

$$
e(\widehat{X}) \leq c\left(\left(\ln h^{-1}\right)^{1 / 2}\left(h^{1 / 2}+h^{3 / 2}\left\|\sigma_{*}^{-3}\right\|_{\infty}\right)+h^{1 / 2}\left\|\sigma_{*}^{-1}\right\|_{\infty}\right) .
$$

In fact, $e(\widehat{X})$ may get arbitrarily large for fixed $h$, if $\sigma$ gets small at some point. Therefore we have introduced the additional term $h \ln h^{-1}$ in the definition of the step size (8). Hereby,

$$
\begin{aligned}
e(\widehat{X}) & \leq c\left(\left(\ln h^{-1}\right)^{1 / 2}\left(h^{1 / 2}+\left(h \ln h^{-1}\right)^{3 / 2}\right)+\left(h \ln h^{-1}\right)^{1 / 2}\right) \\
& \leq c\left(h \ln h^{-1}\right)^{1 / 2}
\end{aligned}
$$

holds uniformly without any lower bound for $\sigma$.

Recall that all constants $c$ do only depend on $K_{1}, \ldots, K_{6}$ and $q, d$ and $m$. Therefore the bounds from Theorem 1 hold uniformly on the class of systems of equations (4) with properties (A)-(C).

4.4. Proof of Theorem 2. Consider an arbitrary sequence of methods $\bar{X}^{n}$ based on $X(0)$ and $n$ observations of $W$. A formal definition of methods of this kind is given in Section 2. In view of Theorem 1 it suffices to show that

$$
\liminf _{n \rightarrow \infty}(n / \ln n)^{1 / 2} e\left(\bar{X}^{n}\right) \geq 1 / \sqrt{2}\left\|\sigma_{*}\right\|_{2} .
$$

The idea of the proof reads as follows. From $\bar{X}^{n}$ we derive an approximation $V^{n}$ of $V$ such that the error of $\bar{X}^{n}$ is essentially determined by $V-V^{n}$. This is achieved by introducing additional equidistant points

$$
\tau_{l}^{n}=l / k_{n}
$$

for $l=1, \ldots, k_{n}$. Now, $V^{n}$ is defined by

$$
V^{n}=\bar{X}^{n}-X(0)-\widehat{Y}^{n}+\widehat{Z}^{n}
$$

where $\widehat{Y}^{n}$ and $\widehat{Z}^{n}$ are determined by (10) with respect to the discretization (23). Note that $V^{n}$ uses at most $m_{n}=n+k_{n}$ observations of $W$. We choose $k_{n}=\left\lceil n /(\ln n)^{1 / 2}\right\rceil$. Clearly $\lim _{n \rightarrow \infty} m_{n} / n=1$. Observing Lemma 1 we obtain

$$
e\left(\bar{X}^{n}\right) \geq\left(E\left(\left\|V-V^{n}\right\|_{\infty}^{q}\right)\right)^{1 / q}-c k_{n}^{-1 / 2}
$$


and $\lim _{n \rightarrow \infty}(n / \ln n) / k_{n}=0$ implies

$$
\liminf _{n \rightarrow \infty}(n / \ln n)^{1 / 2} e\left(\bar{X}^{n}\right) \geq \liminf _{n \rightarrow \infty}\left(m_{n} / \ln m_{n}\right)^{1 / 2}\left(E\left(\left\|V-V^{n}\right\|_{\infty}^{q}\right)\right)^{1 / q} .
$$

We derive a lower bound for $E\left(\left\|V-V^{n}\right\|_{\infty}^{q}\right)$. Since $V$ and $X(0)$ are independent by assumption (C), we may assume that $V^{n}$ does not use the initial value $X(0)$. The process $V$ is Gaussian. Approximating $V$ in $L_{\infty}$-norm from finitely many observations of $V$ defines a linear problem with a Gaussian measure, and for problems of this kind, adaptive selection of the observation points does not help; see Traub, Wasilkowski, and Woźniakowski [(1988), Chapter 6.6.3]. Hence we may assume that $V^{n}$ uses the values of $W$ at fixed points

$$
0<\xi_{1}<\cdots<\xi_{m_{n}}=1 \text {. }
$$

Then we have

$$
\begin{aligned}
E\left(\left\|V-V^{n}\right\|_{\infty}^{q}\right) & \geq E\left(\max _{1 \leq i \leq d}\left\|V_{i}-E\left(V_{i} \mid W\left(\xi_{1}\right), \ldots, W\left(\xi_{m_{n}}\right)\right)\right\|_{\infty}^{q}\right) \\
& =E\left(\|\sigma(W-\tilde{W})\|_{\infty}^{q}\right),
\end{aligned}
$$

where $\widetilde{W}$ denotes the piecewise linear interpolation of $W$ with respect to the points $(25)$.

For $l=1, \ldots, n$ there exists $i_{l} \in\{1, \ldots, d\}$ such that

$$
\sigma_{i_{l}}\left(\xi_{l-1}\right)=\sigma_{*}\left(\xi_{l-1}\right),
$$

and therefore

$$
\max _{\xi_{l-1} \leq t \leq \xi_{l}}\left|\sigma_{i_{l}}(t)-\sigma_{*}(t)\right| \leq c \max _{1 \leq l \leq m_{n}}\left(\xi_{l}-\xi_{l-1}\right) \leq c k_{n}^{-1} .
$$

by (23) and (B). Consequently,

$$
\begin{aligned}
& \left(E\left(\|\sigma(W-\tilde{W})\|_{\infty}^{q}\right)\right)^{1 / q} \\
& \quad \geq\left(E\left(\max _{1 \leq l \leq m_{n}} \max _{\xi_{l-1} \leq t \leq \xi_{l}}\left|\sigma_{i_{l}}(t)\left(W_{1}-\tilde{W}_{1}\right)(t)\right|^{q}\right)\right)^{1 / q} \\
& \quad \geq\left(E\left(\left\|\sigma_{*}\left(W_{1}-\tilde{W}_{1}\right)\right\|_{\infty}^{q}\right)\right)^{1 / q}-c k_{n}^{-1} .
\end{aligned}
$$

Moreover,

$$
\lim _{n \rightarrow \infty}\left(m_{n} / \ln m_{n}\right)^{1 / 2} k_{n}^{-1}=0 .
$$

Combining (24) and (26)-(28) we conclude that

$$
\begin{aligned}
& \liminf _{n \rightarrow \infty}(n / \ln n)^{1 / 2} e\left(\bar{X}^{n}\right) \\
& \quad \geq \liminf _{n \rightarrow \infty}\left(m_{n} / \ln m_{n}\right)^{1 / 2}\left(E\left(\left\|\sigma_{*}\left(W_{1}-\widetilde{W}_{1}\right)\right\|_{\infty}^{q}\right)\right)^{1 / q} .
\end{aligned}
$$


Now we apply a result of Müller-Gronbach and Ritter [(1997), Theorem 1] which provides an asymptotic lower bound for $E\left(\left\|\sigma_{*}\left(W_{1}-\widetilde{W}_{1}\right)\right\|_{\infty}^{q}\right)$ for every discretization (25). We obtain

$$
\liminf _{n \rightarrow \infty}(n / \ln n)^{1 / 2} e\left(\bar{X}^{n}\right) \geq\left\|\sigma_{*}\right\|_{2} 2^{-1 / 2} .
$$

This completes the proof of Theorem 2 .

\section{APPENDIX}

Consider a stochastic differential equation (3) and a fixed discretization (5). An approximation to the solution $X$ of (3) is given by $\breve{X}_{i}(0)=X_{i}(0)$ and

$$
\begin{aligned}
\breve{X}_{i}(t)= & \breve{X}_{i}\left(\tau_{l-1}\right)+\int_{\tau_{l-1}}^{t} a_{i}\left(\tau_{l-1}, \breve{X}\left(\tau_{l-1}\right)\right) d s \\
& +\sum_{j=1}^{m} \int_{\tau_{l-1}}^{t} \sigma_{i, j}\left(\tau_{l-1}, \breve{X}\left(\tau_{l-1}\right)\right) d W_{j}(s),
\end{aligned}
$$

where $\left.t \in] \tau_{l-1}, \tau_{l}\right]$ and $i=1, \ldots, d$. Bouleau and Lépingle [(1994), page 276] present the following upper bound for the error of $\dot{X}$ in the case of constant step size. A straightforward modification of the proof of Theorem B.1.4 in Bouleau and Lépingle (1994) extends the result to the case of variable step size. We use $\left(\mathrm{A}^{\prime}\right)$ to denote the condition that is obtained by replacing $a$ by $\sigma$ in (A).

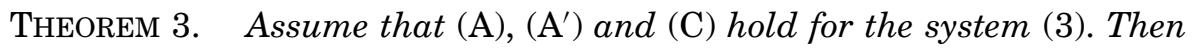

$$
\left(E\left(\|X-\breve{X}\|_{\infty}^{q}\right)\right)^{1 / q} \leq c \Delta_{\max }^{1 / 2} .
$$

The constant $c$ only depends on $q, d, m$, and on the constants from (A), $\left(\mathrm{A}^{\prime}\right)$ and $(\mathrm{C})$.

Note that (31) coincides with the Euler scheme at the discretization points $\tau_{l}$ and satisfies a stochastic differential equation with frozen coefficients on each interval $\left[\tau_{l-1}, \tau_{l}\right]$. Complete knowledge of a trajectory of $W$ is needed to generate the corresponding trajectory of $\breve{X}$. Therefore $\breve{X}$ is not an implementable numerical scheme for the global approximation of $X$. See Cambanis and $\mathrm{Hu}(1996)$ for a detailed asymptotic analysis of $\breve{X}$.

Since $\breve{X}\left(\tau_{l}\right)=\widehat{X}\left(\tau_{l}\right)$ for $l=1, \ldots, n$, we immediately obtain

$$
\left(E\left(\max _{1 \leq i \leq d} \max _{1 \leq l \leq n}\left|X_{i}\left(\tau_{l}\right)-\widehat{X}_{i}\left(\tau_{l}\right)\right|^{q}\right)\right)^{1 / q} \leq c \Delta_{\max }^{1 / 2}
$$

from Theorem 3 . 
Consider a sequence of independent Brownian bridges $B_{l}$ on the interval $T$ with $B_{l}(0)=B_{l}(1)=0$.

LEMMA 3. Let $\alpha, \alpha_{1, n}, \ldots, \alpha_{n, n}>0$ such that

$$
\lim _{n \rightarrow \infty} \max _{1 \leq l \leq n} \alpha_{l, n}=\alpha
$$

and

$$
\liminf _{n \rightarrow \infty} \frac{\#\left\{l: \alpha_{l, n} \geq \alpha-\varepsilon\right\}}{n}>0
$$

for every $\varepsilon>0$. Then

$$
\lim _{n \rightarrow \infty} \int_{\left(\alpha^{2} / 2\right)^{q / 2}}^{\infty} P\left(\max _{1 \leq l \leq n}\left(\alpha_{l, n}(\ln n)^{-1 / 2}\left\|B_{l}\right\|_{\infty}\right)^{q}>u\right) d u=0
$$

and

$$
\lim _{n \rightarrow \infty} P\left(\max _{1 \leq l \leq n}\left(\alpha_{l, n}(\ln n)^{-1 / 2}\left\|B_{l}\right\|_{\infty}\right)^{q}>u\right)=1
$$

for $0 \leq u<\left(\alpha^{2} / 2\right)^{q / 2}$.

See Ritter (1990) for a proof of Lemma 3 in the particular case $\alpha=\alpha_{l, n}$. The lemma immediately yields the following fact.

COROLLARY 1. Under the assumptions of Lemma 3 we have

$$
\lim _{n \rightarrow \infty}(\ln n)^{-1 / 2}\left(E\left(\max _{1 \leq l \leq n}\left(\alpha_{l, n}\left\|B_{l}\right\|_{\infty}\right)^{q}\right)\right)^{1 / q}=\alpha 2^{-1 / 2} .
$$

\section{REFERENCES}

Bouleau, N. and LÉPINGle, D. (1994). Numerical Methods for Stochastic Processes. Wiley, New York.

CAmbanis, S. and Hu, Y. (1996). Exact convergence rate of the Euler-Maruyama scheme, with application to sampling design. Stochastics Stochastics Rep. 59 211-240.

FAURE, O. (1992). Simulation du mouvement brownien et des diffusions. Thèse, ENPC, Paris.

GAINES, J. G. and Lyons, T. J. (1997). Variable step size control in the numerical solution of stochastic differential equations. SIAM J. Appl. Math. 57 1455-1484.

Hofmann, N., MÜlleR-Gronbach, T. and RItTer, K. (1999a). Optimal approximation of stochastic differential equations by adaptive step-size control. Math. Comp. To appear.

Hofmann, N., Müller-Gronbach, T. and RitTer, K. (1999b). The optimal discretization of stochastic differential equations. Preprint A/16/99, Fachbereich Mathematik und Informatik, FU Berlin.

MAUTHNER, S. (1998). Step size control in the numerical solution of stochastic differential equations. Mathematische Preprintreihe, Technische Univ. Darmstadt 1972.

MülleR-Gronbach, T. and RitTER, K. (1997). Uniform reconstruction of Gaussian processes. Stochastic Processes Appl. 69 55-70. 
NEWTON, N. J. (1990). An efficient approximation for stochastic differential equations on the partition of symmetrical first passage times. Stochastics Stochastics Rep. 29 227-258.

RITTER, K. (1990). Approximation and optimization on the Wiener space. J. Complexity 6 337-364.

Traub, J. F., Wasilkowski. G. W. and Woźniakowski, H. (1988). Information-Based Complexity. Academic Press, New York.

N. HOFMANN

MATHEMATISCHES INSTITUT

UNIVERSITÄT ERLANGEN-NÜRNBERG

BISMARCKSTRASSE $11 / 2$

91054 ERLANGEN

GERMANY

E-MAIL: hofmann@mi.uni-erlangen.de
T. MÜLLER-GRONBACH

MATHEMATISCHES InSTITUT

FREIE UNIVERSITÄT BERLIN

ARMINALLEE 2-6

14195 BERLIN

GERMANY

E-MAIL: gronbach@math.fu-berlin.de

K. RITTER

FAKULTÄT FÜR MATHEMATIK UND INFORMATIK

UNIVERSITÄT PASSAU

INNSTR. 33

94032 PASSAU

GERMANY

E-MAIL: klaus.ritter@fmi.uni-passau.de 